\title{
COUNTEREXAMPLES IN BEST APPROXIMATION
}

\author{
S. J. POREDA
}

\begin{abstract}
Several counterexamples for approximation to continuous functions by polynomials are given. One example shows that the points of maximum deviation of a continuous real valued function on an interval from its polynomial of degree $n$ of best uniform approximation can lie on any monotone sequence contained in that interval for infinitely many $n$.
\end{abstract}

1. Notation and definitions. For a complex valued function $f$ defined on a set $E$ in the complex plane let $\|f\|_{E}=\sup _{z \in E}|f(z)|$. If $E$ is compact and $f$ is continuous on $E$, then for each $n \in Z^{+}=\{0,1,2, \ldots\}$ there exists a unique polynomial $p_{n}$ of degree $n$ such that $\left\|f-p_{n}\right\|_{E} \leqslant\left\|f-q_{n}\right\|_{E}$ for all polynomials $q_{n}$ of degree $n$. The polynomial $p_{n}$ is called the polynomial of degree $n$ of best uniform approximation to $f$ on $E$.

2. Extremal points. If $f$ is a real valued continuous function on the real interval $[a, b], n \in Z^{+}$, and $p_{n}$ is the polynomial of degree $n$ of best uniform approximation to $f$ on $[a, b]$, then there exist at least $n+2$ points on $[a, b]$ where $\left|f-p_{n}\right|$ attains its maximum. Let the set of all such points be denoted by $\xi_{n}(f)$ and let $\xi(f)=\cup_{n=0}^{\infty} \xi_{n}(f)$. An immediate question pertains to the distribution of $\xi(f)$ on $[a, b]$. We shall now see that by applying a theorem due to Kadec [1] $\xi(f)$ can be shown to be dense (in a straightforward manner) in $[a, b]$.

THEOREM (KADEC). Let $f(t)$ be a continuous function on $[0, \pi], S_{n}(t)$ the even trigonometric polynomial of degree $n$ of best uniform approximation to $f(t)$ on $[0, \pi]$ and $0 \leqslant t_{0}^{n}<t_{1}^{(n)}<\cdots<t_{n+1}^{(n)} \leqslant \pi$ be $n+2$ points where $f(t)-S_{n}(t)$ assumes, with alternating signs, its maximum absolute value. If we set

$$
\Delta_{n}=\max _{0 \leqslant k \leqslant n+1}\left|t_{k}^{(n)}-\pi k /(n+1)\right|, \quad n=0,2, \ldots,
$$

it then follows that

$$
\varliminf_{n \rightarrow \infty} \Delta_{n} \cdot n^{1 / 2-\varepsilon}=0 \text { for any } \varepsilon>0 .
$$

Corollary. Let $f,[a, b]$ and $\xi(f)$ be as before. Then $\xi(f)$ is dense in $[a, b]$.

Proof. This proof follows directly from Kadec's Theorem if we use the transformation $u=\cos t$ and $x=(b-a) u+a$.

Received by the editors April 14, 1975.

AMS (MOS) subject classifications (1970). Primary 41A10.

Key words and phrases. Best uniform polynomial approximation.

(1) American Mathematical Society 1976 
In light of the previous corollary, it is interesting to find that the extremal points $\xi_{n}(f)$ need not be "spread" out for all $n$ and as a consequence, $: t$ is not

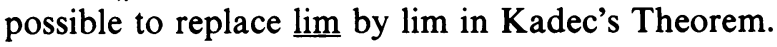

THEOREM 1. Given a monotone sequence $\left\{x_{k}\right\}_{k=1}^{\infty}, \lim _{k \rightarrow \infty} x_{k}=x_{\infty}$ and $\left[x_{0}, x_{\infty}\right] \subset(a, b)$ there exists a function $f$ continuous on $[a, b]$ such that $\xi_{n}(f)$ $\subset\left\{x_{k}\right\}_{k=0}^{\infty}$ for infinitely many $n$.

The proof of this theorem requires use of a theorem due to Wolibner [6] which we shall state here as a lemma.

Lemma 1 (WolibNer). Given $a \leqslant t_{1}<t_{2}<\cdots<t_{n} \leqslant b$ and real numbers $\left\{\omega_{i}\right\}_{i=1}^{n}$ such that $\omega_{i} \neq \omega_{i+1}$ for $i=1,2, \ldots, n-1$, there exists a polynomial $p(x)$ such that,

(1) $p\left(t_{i}\right)=\omega_{i}$ for $i=1,2, \ldots, n$, and

(2) $p(x)$ is monotone on $\left[t_{i}, t_{i+1}\right]$ for $i=1,2, \ldots, n-1$.

Proof of Theorem 1. Let $\left\{x_{k}\right\}_{k=0}^{\infty}, x_{\infty}$ and $[a, b]$ be as in the statement of our theorem and let $Q_{n_{i}}(x)$ be a polynomial such that;

(i) $Q_{n_{i}}\left(\right.$ a) $=-1 / 2^{i+1}$,

(ii) $Q_{n_{i}}\left(x_{0}\right)=1 / 2^{i+1}$,

(iii) $Q_{n_{i}}\left(x_{k}\right)=(-1)^{k} / 2^{i}$ for $k=1,2, \ldots, 2 n_{i}+2$,

(iv) $Q_{n_{i}}\left(x_{2 n_{i}+3}\right)=(-1)^{2 n_{i}+3} / 2^{i+1}$,

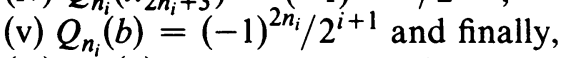

(vi) $Q_{n_{i}}(x)$ is monotone on the intervals $\left[a, x_{0}\right],\left[x_{k}, x_{k+1}\right]$ for $k=1,2, \ldots$, $2 n_{i}+3$, and $\left[x_{2 n_{i}+3}, b\right]$.

To define $n_{i}$, set $n_{1}=2$ and $n_{i+1}$ the degree of $Q_{n_{i}}$ Clearly the degree of $Q_{n_{i}}$ is greater than $n_{i}$ since it has more than $n_{i}$ zeros. Now set $f(x)=\sum_{i=1}^{\infty} Q_{n_{i}}(x)$ and let $P_{n_{i}}(x)=\sum_{j=1}^{i-1} Q_{n_{j}}(x)$. Clearly, $P_{n_{i}}(x)$ is a polynomial of degree $n_{i}$; $f\left(x_{k}\right)-P_{n_{i}}\left(x_{k}\right)=(-1)^{k} / 2^{i-1}$, for $k=1,2, \ldots, 2 n_{i}+2$, and $\left\|f-P_{n_{i}}\right\|_{[a, b]}$ $=1 / 2^{i-1}$ for $i=1,2, \ldots$.

Now [7, p. 20] since the polynomial $p_{n}(x)$ of degree $n$ of best uniform approximation to a continuous function $f(x)$ on the interval $[a, b]$ is characterized by the existence of $n+2$ points $\left\{x_{k}\right\}_{k=1}^{n+2}, a \leqslant x_{k}<x_{k+1} \leqslant b, k=1,2$, $\ldots, n+1$, where the deviation $f(x)-p_{n}(x)$ attains its maximum on $[a, b]$ with alternating sign, it follows that, as constructed, $P_{n_{i}}(x)$ is the polynomial of degree $n_{i}$ of best uniform approximation to $f(x)$ on $[a, b]$ and $\xi_{n_{i}}(f)$ $\subset\left[x_{0}, x_{\infty}\right]$ for $i=1,2, \ldots$.

REMARK 1. As constructed, $f(x)$ has the property that

$$
\left\|f-P_{n_{i}}\right\|_{[a, b]} \geqslant 2\left\|f-P_{n_{i}}\right\|_{\left[a, x_{0}\right] \cup\left[x_{\infty}, b\right]}, \quad \text { for } i=1,2, \ldots,
$$

where $P_{n_{i}}$ is the polynomial of degree $n_{i}$ of best uniform approximation to $f$ on $[a, b]$, and $n_{i}$ is as in our construction.

3. Uniform continuity of best uniform polynomial approximations. If $f$ is a continuous, complex valued function defined on a compact set $E$ in the complex plane and if $p_{n}$ is the polynomial of degree $n, n \in Z^{+}$, of best uniform approximation to $f$ on $E$, then [3] there exists a least constant $M_{n}(f)>0$, which depends on $f$ and $n$, such that 


$$
\left\|p_{n}-q_{n}\right\|_{E} \leqslant M_{n}(f)\left[\left\|f-q_{n}\right\|_{E}-\left\|f-p_{n}\right\|_{E}\right]^{1 / 2}
$$

for every polynomial $q_{n}$ of degree $n$. This is sometimes called the "strong uniqueness theorem", for it implies that if $q_{n}$ approximates $f$ almost as well as $p_{n}$ does, then $q_{n}$ is almost equal to $p_{n}$. In the case where $f$ is real and $E$ is an interval, we have the same result except that we can replace the exponent $\frac{1}{2}$ by 1 in the expression (3.1).

Two questions arise here. The first is whether for fixed $f$, the sequence $\left\{M_{n}(f)\right\}_{n=0}^{\infty}$ is bounded. The second is whether for $n$ and $E$ fixed, the set $\left\{M_{n}(f)\right\}_{f \in B(E)}$ is bounded where $B(E)$ denotes the unit ball of $C(E)$, the space of continuous functions on $E$. The purpose of this section is to show that neither of these sets need be bounded. For the first question, we give one example for the real case and one for the complex case. For the second question, we give an example in the real case.

THEOREM 2. For every nonempty real interval $[a, b]$ there exists a function $f$ continuous on $[a, b]$ such that $\lim _{n \rightarrow \infty} M_{n}(f)=\infty$.

Proof. Let $f(x)$ be the function that we constructed in the proof of Theorem 1 where $x_{0}$ and $x_{\infty}$ are chosen so that

$$
x_{0}-a=x_{\infty}-x_{0}=b-x_{\infty}=2 \rho .
$$

Let $\bar{x}=\left(x_{0}+x_{\infty}\right) / 2$ and for every $n_{i}, i=0,1,2, \ldots$, let

$$
q_{n_{i}}(x)=P_{n_{i}}(x)+\left[\left\|f-P_{n_{i}}\right\|_{[a, b]} / 2\right][(x-\bar{x}) / 3 \rho]^{n_{i}}
$$

where $P_{n_{i}}$ is the polynomial of degree $n_{i}$ of best uniform approximation to $f$ on $[a, b]$, and $n_{i}$ is as in our construction.

$$
\left\|q_{n_{i}}-P_{n_{i}}\right\|_{[a, b]}=\left\|f-P_{n_{i}}\right\|_{[a, b]} / 2
$$

and

$$
\begin{aligned}
\left\|f-q_{n_{i}}\right\|_{\left[a, x_{0}\right] \cup\left[x_{\infty}, b\right]} & \leqslant\left\|f-P_{n_{i}}\right\|_{\left[a, x_{0}\right] \cup\left[x_{\infty}, b\right]}+\left\|P_{n_{i}}-q_{n_{i}}\right\|_{\left[a, x_{0}\right] \cup\left[x_{\infty}, b\right]} \\
& \leqslant\left\|f-P_{n_{i}}\right\|_{[a, b]},
\end{aligned}
$$

with the latter following from Remark 1. Hence, since $p_{n_{i}}$ is the best approximation to $f,\left\|f-q_{n_{i}}\right\|_{[a, b]}=\left\|f-q_{n_{i}}\right\|_{\left[x_{0}, x_{\infty}\right]}$. Now by returning to the definition of $q_{n_{i}}$ we see that

$$
\left\|f-q_{n_{i}}\right\|_{[a, b]}-\left\|f-P_{n_{i}}\right\|_{[a, b]}<\frac{1}{2}\left\|f-P_{n_{i}}\right\|_{[a, b]} / 3^{n_{i}},
$$

and so $M_{n_{i}}(f) \geqslant\left\|q_{n_{i}}-P_{n_{i}}\right\|_{[a, b]} /\left[\left\|f-q_{n_{i}}\right\|_{[a, b]}-\left\|f-P_{n_{i}}\right\|_{[a, b]}\right]>3^{n_{i}}$ which implies that $\lim _{i \rightarrow \infty} M_{n_{i}}(f) \stackrel{\infty}{=}$.

In the complex case an example of a function for which $\left\{M_{n}(f)\right\}_{n=0}^{\infty}$ is not bounded is much easier to find. In fact, as we shall see, every function that is badly approximable [4] on a circle, that is, every continuous function which has a constant modulus and whose image has a negative winding number (with respect to the origin), has this property.

THEOREM 3. If $f(z)$ is badly approximable on the unit circle $U=\{|z|=1\}$ then $\lim _{n \rightarrow \infty} M_{n}(f)=\infty$. 
Proof of Theorem 3. Suppose $f$ is badly approximable on $U$. We may assume that $\|f\|_{U}=1$. Choose any $\varepsilon>0$ and let $\Gamma$ be a closed Jordan curve that contains the points $z=-\varepsilon / 3$ and $z=1$ such that:

(i) the interior of $\Gamma$ contains $z=0$;

(ii) $\Gamma \subset[\{|z| \leqslant 1\} \cap\{|\operatorname{Im} z| \leqslant \varepsilon / 3\}]$; and

(iii) $[\Gamma \cap\{\operatorname{Re} z \leqslant 0\}] \subset\{|z| \leqslant \varepsilon / 3\}$.

For instance, $\Gamma$ can be chosen to be an ellipse. Let $h(z)$ be a conformal map of the disc $\{|z|<1\}$ onto the interior of $\Gamma$ such that $h(0)=0$.

Now since $f$ is continuous and of constant modulus on $U$, there exists [5] a finite Blaschke product $B(z)$ of the form;

$$
B(z)=e^{i \mu} \prod_{k=1}^{m}\left(\frac{1-\bar{a}_{k} z}{z-a_{k}}\right) \prod_{j=1}^{l}\left(\frac{z-b_{j}}{1-\bar{b}_{j} z}\right)
$$

where $\left|a_{k}\right|<1$ for $k=1,2, \ldots, m$ and $\left|b_{j}\right|<1$ for $j=1,2, \ldots, l$ and such that $\|f-B\|_{U}<\varepsilon / 3$.

Set

$$
g(z)=B(z) h\left(\prod_{k=1}^{m}\left(\frac{z-a_{k}}{1-\bar{a}_{k} z}\right)\right) .
$$

Since $h$ vanishes at $z=0$, the function $g(z)$ is analytic in $\{|z|<1\}$ (except for removable singularities at $\left.a_{k}, k=1,2, \ldots, m\right)$ and continuous in $\{|z| \leqslant 1\}$. Consequently there exists a polynomial $q_{n}(z)$ of some degree $n$ such that $\left\|g-q_{n}\right\|_{U}<\varepsilon / 3$. Thus we can write

$$
\begin{aligned}
\left\|f-q_{n}\right\|_{U} & \leqslant\|f-B\|_{U}+\|B-g\|_{U}+\left\|g-q_{n}\right\|_{U} \\
& \leqslant \varepsilon / 3+(1+\varepsilon / 3)+\varepsilon / 3=1+\varepsilon,
\end{aligned}
$$

since

$$
\|B-g\|_{U}=\left\|1-h\left(\prod_{k=1}^{m}\left(\frac{z-a_{k}}{1-\bar{a}_{k} z}\right)\right)\right\|_{U}=\max _{z \in \Gamma}|1-z|=1+\varepsilon / 3 .
$$

Now since the polynomial of degree $n$ (or of any degree) of best uniform approximation to $f$ on $U$ is identically zero, it follows that

$$
M_{n}(f) \geqslant\left\|q_{n}\right\|_{U} /\left[\left\|f-q_{n}\right\|_{U}-\|f\|_{U}\right]^{1 / 2} \geqslant(1-\varepsilon / 3) / \varepsilon^{1 / 2} .
$$

Since $\varepsilon$ was arbitrarily chosen, we see that $\left\{M_{n}(f)\right\}_{n=0}^{\infty}$ is not bounded and, in fact, that $\lim _{n \rightarrow \infty} M_{n}(f)=\infty$. Note that had we replaced the exponent $\frac{1}{2}$ in expression (3.1) with some $\beta, 0<\beta<\frac{1}{2}$ and thus defined a new constant, say, $M_{n}^{\beta}(f)$, then the techniques used in our proof are still applicable and we will get $\lim _{n \rightarrow \infty} M_{n}^{\beta}(f)=\infty$.

REMARK 2. Let $B$ denote the unit ball of $C([0,1])$, the space of continuous real valued functions on $[0,1]$. Then for $n \geqslant 1$, the set $\left\{M_{n}(f)\right\}_{f \in B}$ is not bounded. We demonstrate this by noting that for any fixed $f \in B, M_{n+1}(f)$ $\geqslant M_{n}(f)$, so it is sufficient to show that $\left\{M_{1}(f)\right\}_{f \in B}$ is not bounded.

For any $0<\varepsilon<1$, define a function $f(x)$ continuous on $[0,1]$ so that (i) $f(0)=1$, 
(ii) $f(\varepsilon / 2)=-1+\varepsilon / 2$,

(iii) $f(\varepsilon)=1+\varepsilon$,

(iv) $f(1)=0$ and

(v) $f(x)$ is linear between these nodes.

The polynomial of degree 1 of best uniform approximation to $f$ on $[0,1]$ will then be $p_{1}(x)=x$ and $\left\|f-p_{1}\right\|_{[0,1]}=1$. Now since $\|f\|_{[0,1]}=1+\varepsilon$, if we let $q_{1} \equiv 0$, we have that $M_{1}(f) \geqslant\left\|p_{1}\right\|_{[0,1]} / \varepsilon=1 / \varepsilon$. The function $f(x) /(1+\varepsilon)$ $\in B$ and so $M_{1}(f(x) /(1+\varepsilon)) \geqslant 1 / \varepsilon(1+\varepsilon)$ which implies our theorem since $\varepsilon$ was chosen arbitrarily.

4. Open problems. In light of Kadec's Theorem (Theorem 1) and Theorem 3 , it seems likely that more can be said about the sequence $\left\{M_{n}(f)\right\}_{n=1}^{\infty}$. For instance, it might be interesting to characterize those functions, in both the real and complex cases, for which the sequence $\left\{M_{n}(f)\right\}_{n=1}^{\infty}$ is bounded.

\section{REFERENCES}

1. M. I. Kadec, On the distribution of points of maximum deviation in the approximation of continuous functions by polynomials, Uspehi Mat. Nauk 15 (1960), no. 1 (91), 199-202; English transl., Amer. Math. Soc. Transl. (2) 26 (1963), 231-234. MR 22 \#3920; 27 \# 1750.

2. V. I. Smirnov and N. A. Lebedev, The constructive theory of functions of a complex variable, "Nauka", Moscow, 1964; English transl., Functions of a complex variable: Constructive theory, M.I.T. Press, Cambridge, Mass., 1968. MR 30 \#2152; 37 \# 5369.

3. H. S. Shapiro, Topics in approximation theory, Springer-Verlag, Berlin and New York, 1971, p. 26.

4. S. J. Poreda, A characterization of badly approximable functions, Trans. Amer. Math. Soc. 169 (1972), 249-256. MR 46 \#5636.

5. - Approximation by $\delta$-polynomials, Siam J. Numer. Anal. 10 (1973), 50-54. MR 47 \#5265.

6. W. Wolibner, Sur un polynome d'interpolation, Colloq. Math. 2 (1951), 136-137. MR 13, 343.

7. G. Meinardus, Approximation of functions: Theory and numerical methods, Springer Tracts in Natural Philosophy, vol. 13, Springer-Verlag, New York, 1967. MR 36 \#571.

Department of Mathematics, Clark University, Worcester, Massachusetts 01610 